\title{
DUAL-WAVELENGTH HIGH-SPECTRAL-RESOLUTION LIDAR FOR PROFILING OPTICAL PROPERTIES OF AEROSOL AND CLOUD
}

\author{
Xue Shen ${ }^{1}$, Nanchao Wang ${ }^{1}$, *Dong Liu ${ }^{1}$, Da Xiao ${ }^{1}$, Yuhang Rong ${ }^{1}$, Tianfen Zhong ${ }^{1}$, Chong Liu ${ }^{1}$, \\ Yupeng Zhang ${ }^{1}$, Yudi Zhou ${ }^{1}$, Sijie Chen ${ }^{1}$ \\ ${ }^{1}$ State Key Laboratory of Modern Optical Instrumentation, College of Optical Science and Engineering, \\ Zhejiang University, Hangzhou 310027, China \\ Email:*liudongopt@zju.edu.cn
}

\begin{abstract}
A dual-wavelength high-spectral-resolution lidar (HSRL) based on an iodine absorption filter and a field-widened Michelson interferometer (FWMI) has been developed to profile backscatter and extinction coefficients of aerosols and clouds accurately. This instrument was tested and calibrated on multiple observations in Hangzhou and Zhoushan, respectively, from August 2018 to April 2019. This paper discusses the design and the internal calibration method of the lidar system in detail, with several typical cases of observations and the analysis of these data products. The optical properties of urban aerosols in Hangzhou and the evolvement of clouds in Zhoushan are presented, respectively.
\end{abstract}

\section{INTRODUCTION}

There is a strong need to make more observations on the vertical distribution and optical properties of aerosols and clouds. Both aerosols and clouds play significant roles in influencing the Earth's climate, but our limited understanding of them leads to very large uncertainties in our ability to estimate the climate changes. The vertical distribution and optical properties of clouds contribute significantly to the climate models. As for aerosols, more accurate estimates of their impact on climate require global monitoring of aerosol scattering and absorption during their production and transportation. Furthermore, the vertical distribution of the aerosols can induce aerosol-cloud interaction. Unfortunately, these parameters are difficult to estimate from passive satellite data. On the other hand, Lidar is widely recognized as a necessary component in any strategy to provide the information on aerosol and cloud distribution and optical properties required to address these outstanding issues [1,2].

Using a conventional backscatter lidar, the optical properties cannot be measured directly, only with assumption of the extinction-to-backscatter ratio, the so-called lidar ratio. However, the lidar ratio is a highly variable quantity in atmosphere, so that large errors in the retrieval are expected. Using a high spectral resolution lidar (HSRL) is a good way to solve this problem. HSRL takes advantage of the different spectral broadening of light, backscattered by molecules and aerosols. By means of a narrow bandwidth optical filter the aerosol contribution is separated from the molecular backscatter. Thus, backscatter and extinction coefficients can be measured directly and no assumption about the lidar ratio is required [3]. Furthermore, our preliminary study shows that the parameters such as spectral depolarization ratio and color ratio can be obtained by adding a lidar of another wavelength on the basis of a single-wavelength HSRL, which can reduce the discrimination error of component and intensity of aerosols [4]. Therefore, a dual-wavelength HSRL is of great significance for the determination of the vertical structure and evolution of clouds and aerosols.

This paper introduces the first dual-wavelength HSRL independently developed by Zhejiang University. The system design and calibration method are introduced at first. Some typical results of the initial observation are subsequently presented and analyzed, which demonstrate the system's performance.

\section{METHODOLOGY}

\subsection{The lidar system}

The developed dual-wavelength HSRL system mainly includes transmitting part and receiving part. The transmitter used for the HSRL measurements is a injection seeded $\mathrm{Nd}$ : YAG laser with a narrow-linewidth $\mathrm{CW}$ laser. It has pulse energies of $100 \mathrm{~mJ}$ at $532 \mathrm{~nm}, 150 \mathrm{~mJ}$ at $1064 \mathrm{~nm}$, and a laser repetition rate of $10 \mathrm{~Hz}$. The atmospheric backscatter is collected by means of a $280 \mathrm{~mm}$ Cassegrain telescope. A field stop within 
the focal plane of the telescope limits the field of view to $0.36 \mathrm{mrad}$. Dichroic beam splitters are used to spectrally separate the backscatter signals at $1064 \mathrm{~nm}$ and $532 \mathrm{~nm}$. The $532 \mathrm{~nm}$ subsystem is filtered with an interference filter of $0.3 \mathrm{~nm}$ bandwidth to suppress sunlight, and then use an iodine filter to suppress particle-dominated components for the HSRL measurement. At 1064 $\mathrm{nm}$, a pressure-tuned field-widened Michelson interferometer (FWMI) is used. Figure 1(a) shows the picture of HSRL's receiving system. The entire lidar system is deployed in an experimental container, as Fig. 1(b) shown, which can provide good working conditions for HSRL.

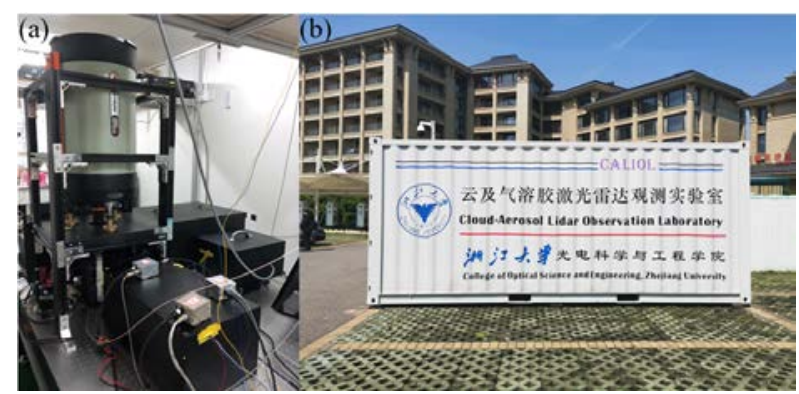

Fig.1. (a) The dual-wavelength high spectral resolution lidar, (b) the experimental container deployed in Zhoushan to accomplish the first field observation.

\subsection{Calibration approach}

In the polarized HSRL, several calibrations should be made before the instrument operation. The first one is the gain ratio of polarization channels. We use a common approach called the ' $\pm 45^{\circ}$ calibration method', i.e., rotating the polarization direction of laser beam with respect to the polarized beam splitter, and recording the signals of the detector in the parallel channel and perpendicular channel [5].The second calibration is on the gain ratio of molecular channel with the background transmittance of narrowband discriminators. We prepare a laser beam has the same properties as the emitted laser but with multiple attenuations of energy, straight into the telescope. The frequency of the laser beam should be scanned on, and the largest ratio of molecular signal to combined signal is the target value. At the same time, the spectrum transmittance of the discriminator can be measured. The third calibration is signal transmittance of Rayleigh and Mie can be calculated with the spectrum transmittance measured. This method was inspired by NASA's calibration method using backscattered signal of hard target [6]. The fifth calibration is the overlap function of the HSRL. Since the HSRL instrument we built cannot observe the atmosphere horizontally, the commonly used 'horizontal calibration' for overlap factor would be unavailable. We add an extra backscatter lidar receiver besides HSRL to measure system's overlap function [7]. This is an ingenious design because there is no need to calibrate the difference between the two systems according to HSRL's algorithm. Overall, lidar calibration is an interesting and challenging topic, which needs more researches to be done in order to improve the retrieval accuracy.

\section{RESULTS}

\subsection{Aerosol optical properties}

Figure 2 shows the retrieval results of depolarization ratio and lidar ratio of aerosol measured in Hangzhou. These images have a temporal resolution of $100 \mathrm{~s}$ and a spatial resolution of $7.5 \mathrm{~m}$. The aerosol layer extends to $3 \mathrm{~km}$, the depolarization ratio value is around 0.1 , and the lidar ratio is around $40 \mathrm{sr}$, which fits the range of urban aerosol optical properties in the literature [4]. 
(a) The Depolarization Ratio (Unit)

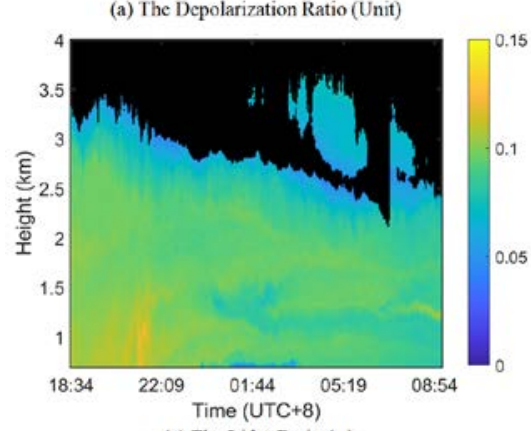

(c) The Lidar Ratio (sr)

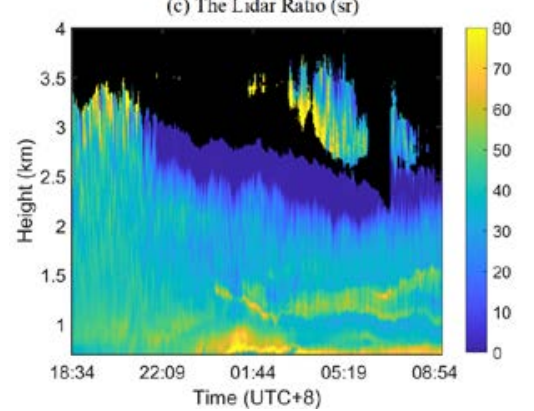

(b) The Depolarization Ratio

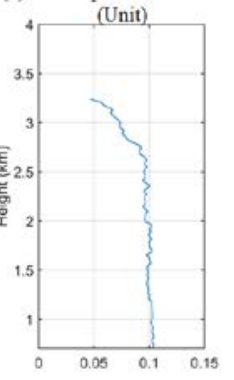

(d) The Lidar Ratio (sc)

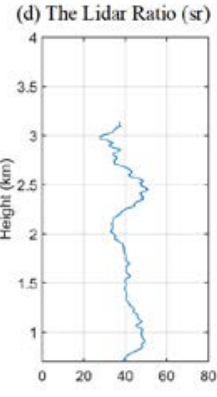

Fig.2. Measured aerosol's depolarization ratio (a \& b) and lidar ratio (c \& d) in Hangzhou from 18:34 pm (UTC + 8), 12 March 2019 to 8:54 am (UTC + 8), 13 March 2019.

\subsection{Cloud optical properties}

Figure 3 shows the retrieval results of the cloud evolution process before a rainfall at Dongshawan beach in Zhoushan. This image has a temporal resolution of $100 \mathrm{~s}$ and a spatial resolution of $7.5 \mathrm{~m}$. As the figure shows, there is a relatively obvious cloud signal below $14 \mathrm{~km}$. As the rain approaches,
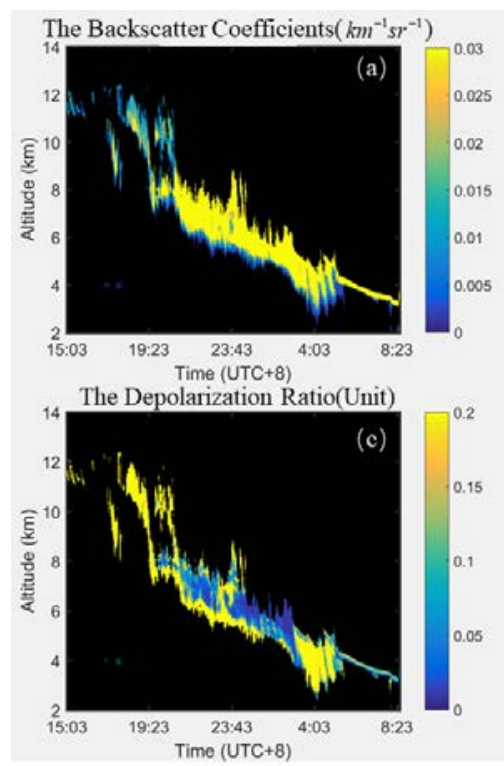

the cloud gradually falls, and the air humidity can be detected to gradually increase. When the rain falls at 8:23 am on the second day, the cloud drops to the vicinity of $3 \mathrm{~km}$, then the lidar system is shut down.
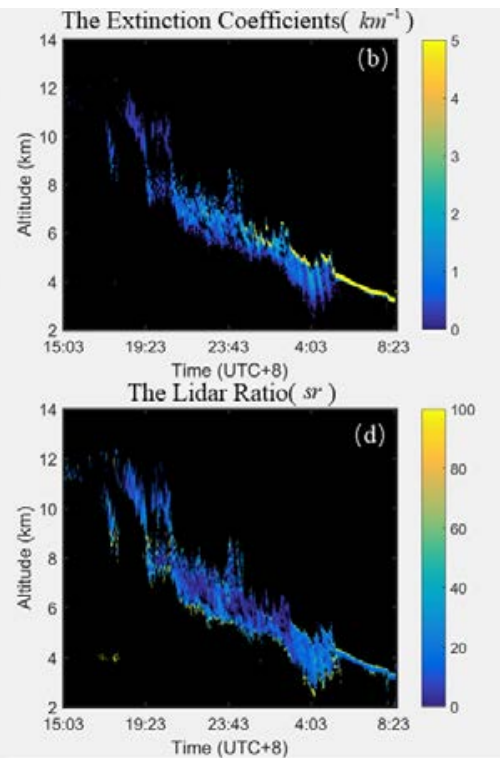

Fig.3. The retrieval results of cloud evolution in Zhoushan from 15:03pm, 15 April 2019 to 8:23am, 16 April 2019. These four pictures are: (a) the backscatter coefficients; (b) the extinction coefficients; (c) the depolarization ratio; (d) the extinction-tobackscatter ratio. 
Figure 3 presents data products for the field experiment, which demonstrate the power of the HSRL for observing the spatial distribution of cloud, quantifying cloud optical parameters, and inferring cloud type. The extensive parameters measured with the high spectral resolution technique are backscatter and extinction coefficients at 532nm, and are shown in Figs. 3(a) and $3(\mathrm{~b})$. The backscatter data is averaged over $100 \mathrm{~s}$ and $7.5 \mathrm{~m}$ in altitude, and the extinction is analyzed over $100 \mathrm{~s}$ and $7.5 \mathrm{~m}$ in altitude. The intensive parameters measured by the HSRL instrument are shown in Figs. 3(c) and 3(d) and include the depolarization ratio at $532 \mathrm{~nm}(100 \mathrm{~s}$, $7.5 \mathrm{~m})$, the extinction-to-backscatter ratio $(\mathrm{Sa})$ $(100 \mathrm{~s}, 7.5 \mathrm{~m})$. As depicted by Fig.3, in the evening of April 15, 2019, rain-producing clouds form below the cirrus clouds, along with the depolarization ratio of cloud layer tends to decrease, and the backscatter coefficient increases gradually [8]. By the morning of April 16, 2019, just before the rain, clouds were gathering around $3 \mathrm{~km}$ above the ground. The backscattering of rainproducing clouds is extremely large, and the lidar ratio is between $15 \mathrm{sr}$ and $20 \mathrm{sr}$. The results shown in Fig. 3 are reasonable. Figure 4 shows the profile of cirrus clouds. At this time, there is a strong backscattering signal at an altitude of 10$12 \mathrm{~km}$. The backscattering coefficient reaches up to $0.02 \mathrm{~km}^{-1} \mathrm{sr}^{-1}$, and the maximum scattering ratio is up to 60 , while the depolarization ratio is as much as 0.3 , the lidar ratio varies from 5 sr to 10 sr, which conforms to the reported range of cirrus clouds' optical properties.
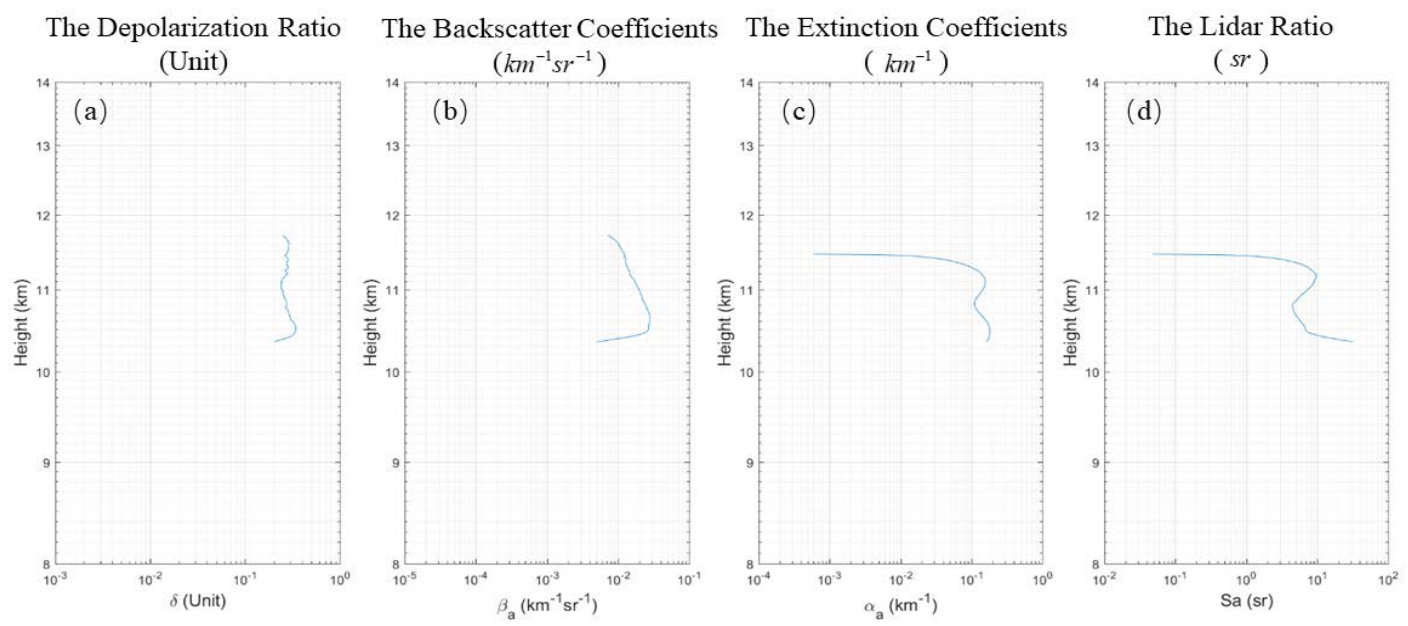

Fig.4. Retrieval profiles of cirrus clouds at an altitude of 10-12km around 18:35pm, 15 April 2019. These four pictures are: (a) the depolarization ratio; (b) the backscatter coefficients; (c) the extinction coefficients; (d) the extinction-to-backscatter ratio.

\section{ACKNOWLEDGEMENTS}

This work was supported by the National Key Research and Development Program of China (2016YFC1400900, 2016YFC0200700); National Natural Science Foundation of China (NSFC) (41775023); Excellent Young Scientist Program of Zhejiang Provincial Natural Science of China (LR19D050001); Fundamental Research Funds for the Central Universities; State Key Laboratory of Modern Optical Instrumentation Innovation Program.

\section{REFERENCES}

[1] Hair J W, Hostetler C A, Cook A L, et al. Applied Optics, 47(36):6734-52 (2008).

[2] Francois-Marie Bréon. Science, 313(5787):623-624 (2006).

[3] Esselborn M, Wirth M, Fix A, et al. Applied Optics, 47(3):346-358 (2008).

[4] Dong Liu, et al. Journal of Applied Remote Sensing, 9(1):096006 (2015).

[5] B. Liu, and Z. E. Wang, Optics express 21, 1458314590 (2013).

[6] S. P. Burton, C. A. Hostetler, et al. Appl. Opt. 57, 6061-6075 (2018)

[7] Juan Luis Guerrero-Rascado, et al. Opt. Express 18, 20350-20369 (2010)

[8] S. Groß, et al. Atmospheric Chemistry and Physics 13.5(2013). 